\begin{tabular}{|c|l|}
\hline Title & A pplication of Cohort A nalysis to Large Terrestrial Mammal Harvest Data \\
\hline Author(s) & Ueno, Mayumi; Matsuishi, Takashi; Solberg, Erling Johan; Saitoh, Takashi \\
\hline Citation & $\begin{array}{l}\text { Mammal Study, 34(2), 65-76 } \\
\text { https://doi.org/40.3106/041.034.0202 }\end{array}$ \\
\hline Issue Date & 2009 \\
\hline Doc URL & http://hdl.handle.net/2115/44226 \\
\hline Type & article \\
\hline File Information & Ueno et al. 2009.pdf \\
\hline
\end{tabular}

Instructions for use 


\title{
Application of cohort analysis to large terrestrial mammal harvest data
}

\author{
Mayumi Ueno $^{1, *}$, Takashi Matsuishi ${ }^{2}$, Erling Johan Solberg ${ }^{3}$ and Takashi Saitoh ${ }^{4}$ \\ ${ }^{1}$ Graduate School of Agriculture, Hokkaido University \\ ${ }^{2}$ Graduate School of Fisheries Sciences, Hokkaido University \\ ${ }^{3}$ Norwegian Institute for Nature Research (NINA) \\ ${ }^{4}$ Field Science Center for Northern Biosphere, Hokkaido University
}

\begin{abstract}
Cohort analysis (also known as virtual population analysis) is a method of population reconstruction from age-specific harvest data. Because cohort analysis requires data over a whole life span to reconstruct a population for a single year, this method is impracticable for longer-lived animals. Three models are routinely combined by fisheries scientists to make cohort analysis more cost effective and to provide real-time estimates of population size; these models may be applied to large terrestrial mammal harvest data. Each model has unique assumptions about hunting mortality rates or age distributions, and the reliability of estimates depends on meeting these assumptions. In this study, we first tested previously used assumptions for these models through an analysis of longterm moose (Alces alces) harvest data, followed by an examination of the robustness of estimates for each moose age class. We developed practical ways to achieve more realistic assumptions for two of three models and showed that meeting these assumptions was more important in estimations of large terrestrial mammal population parameters than for fish population parameters. Therefore, we recommend compliance with assumptions of the three models for more reliable population estimates of large terrestrial mammals.
\end{abstract}

Key words: estimation error, hunting, methodology, population size, virtual population analysis.

Modern wildlife management often depends on good estimates of population size and growth. Although radio-telemetry surveys provide reliable estimates of population sizes via capture-mark-recapture methods (Lebreton et al. 1992), their efficacy depends on the number of individuals animals included in the surveys, making the method time- and labor-intensive in many cases where accuracy of estimation is of importance. On the other hand, large harvest data sets for game species, often collected by age and sex as part of wildlife agency management programs (Skalski and Millspaugh 2002; Conn et al. 2008), are more readily available; under many circumstances, these data can be useful for population estimation.

Cohort analysis (also known as virtual population analysis, VPA) is a method for population reconstruction applied to age-specific harvest data (harvest-at-age). Originally developed in fisheries science (Gulland 1965; Pope 1972), the technique has been applied to commercially harvested fish stocks (bigeye tuna Thunnus obesus,
Nishida and Takeuchi 1999; chub mackerel Scomber japonicus, Yatsu et al. 2002; Pacific cod Gadus macrocephalus, Ueda et al. 2006), aquatic mammals (sperm whale Physeter macrocephalus, Shirakihara 1991; minke whale Balaenoptera acutorostrata, Fujise et al. 2005; minke whale, Mori et al. 2007) and terrestrial mammals (red deer Cervus elaphus, Lowe 1969; white-tailed deer Odocoileus virginiaus, McCullough 1979; marten Martes americana, Fryxell et al. 1999; roe deer Capreolus capreolus, Lowe and Thompson-Schwab 2003). Cohort analysis is based on the principle that individuals born in a particular year (a cohort) will die either from natural causes or from harvesting (i.e., human exploitation) and that mortality may occur at any point in time up to the cohort's oldest age-class. Cohort analysis enables calculation of the number of individuals in a particular ageclass (number-at-age) by aggregating the numbers of animals harvested from that age class $(a)$ to the point when they reach oldest age-class $(A)$; the natural mortality rate is also incorporated into the calculations. By

*To whom correspondence should be addressed.E-mail: mayumi@exfor.agr.hokudai.ac.jp 
combining calculations for various cohorts, population size in each year can be reconstructed. Thus, cohort analysis can be a useful method for population reconstruction when harvest-at-age data are available for each year, and the assumptions of a closed population (no immigration or emigration) and a particular natural mortality rate are fulfilled.

There are, however, two major cost- and time-related problems. One is that a long time series of data is required. To calculate total population size for any previous year, data must span a period extending beyond the lifespan of individuals born in that year. This requirement becomes especially problematic in large terrestrial mammals, such as cervids, which can live up to 20 years (Lowe 1969; Solberg et al. 2000). The second problem, arising from the first, is that it is not possible to calculate recent population sizes. We adapted this original cohort analysis to make it more cost effective and to provide real-time estimates for large terrestrial mammals using harvest data.

This estimation can be achieved by combining three models with the original cohort analysis: (1) a model for age-specific hunting mortality rate in the most recent year, ( $Y$; Model A), (2) a model for the rate of hunting mortality in a composite age class of $(p)$ and the older, (plus-group, $p+$; Model B), and (3) a model for the age distribution in the plus-group (Model C), which has been used routinely in fisheries science (Ueda et al. 2006; Yamaguchi and Matsuishi 2007). Applying Models A, $\mathrm{B}$, and $\mathrm{C}$, number-at-age in year $Y$ and number at age $p+$ in each year can be estimated. Subsequently, the numbers-at-age for any year can computed in the same way as the original cohort analysis. Cohort analysis combining the three models, therefore, is expected to be cost effective and provide real-time estimates for large terrestrial mammals.

However, there has been no cohort analysis for large terrestrial mammals combining these three models. Because each model has particular assumptions regarding rates of hunting mortality or age distribution in the plus-group, the reliability of estimated numbers-at-age will, in turn, depend on meeting the assumptions. The issue needs to be investigated before cohort analysis combining three models can be applied routinely to large terrestrial mammal harvest data.

With a sufficiently long data set, we can reconstruct a population using the original cohort analysis by assuming that catch-at-age for the oldest age-class is equal to number-at-age for that age-class. Then, we can compare calculated number-at-age from the original cohort analysis with number-at-age estimated by modeling to test assumptions and to explore potentially more realistic assumptions. This approach is similar to retrospective analysis (e.g., Shimoda et al. 2006). Pope (1972) demonstrated theoretically that estimates will be more robust when aggregated harvests-at-age make up the greatest proportion of the estimates (represented by the rate of age-specific cumulative hunting mortality). We can therefore examine robustness of the estimates by quantifying the age-specific cumulative hunting mortality rate. We used harvest-at-age data for moose in Norway collected over a sufficiently long period to enable calculation (by the original cohort analysis method) of numberat-age over a period of several years.

To develop a method for large terrestrial mammal population reconstruction, our objectives were to explore ways to meet realistic assumptions for each of the models $\mathrm{A}, \mathrm{B}$, and $\mathrm{C}$, and to determine the extent to which the assumptions affect the robustness of estimated number-at-age. For the first objective, we calculated number-at-age by using the original cohort analysis method, and then tested previously-used assumptions for Models A, B, and C, exploring comparable alternative assumptions for more reliable estimates. As part of this process, we demonstrated a cohort analysis combining three models using limited periods of harvest data, and tested the performance of comparable alternative assumptions. For the second objective, we quantified the agespecific rate of cumulative hunting mortality to examine the robustness of number-at-age estimations.

\section{Material and methods}

Data

We used harvest-at-age data for female moose in Gausdal municipality, Oppland county, southern Norway $\left(61^{\circ} 26^{\prime} \mathrm{N}, 9^{\circ} 83^{\prime} \mathrm{E}\right) . \quad$ In the period $1981-2004,2314$ moose were killed in the area and age was determined for 2235 (96.5\%) (Solberg et al. 2006). The annual proportions of animals within age groups (0 year-old moose was classified as 'young', 1 year-old as 'yearlings', and $\geq 2$ year-old as 'adults') ranged between $87 \%$ and $100 \%$. To correct for missing individuals, the annual number of moose harvested within each age group was multiplied by a factor correcting for the number of missing individuals in the data set. For example, when only $x$ of $y$ adults were harvested and aged in a particular year, the harvest number within each adult age-group for the year was 
multiplied by $y / x$ to correct for the deviation. The study area and harvest-at-age data are more fully described by Solberg et al. $(2002,2006)$.

The following notation was used for analyses: $C_{a, y}$, the corrected number of harvest-at-age $a$ in year $y ; N_{a, y}$, the calculated number of individuals alive at age $a$ in year $y$ based on the original cohort analysis; $F_{a, y}$, the hunting mortality rate at age $a$ in year $y$ based on the calculated number-at-age; $M$, the assumed natural mortality rate. Mortality "rate" in this study was actually represented by a mortality coefficient rather than a rate. Nevertheless, we refer to mortality rate, as this term is in more general use.

\section{Natural mortality rate ( $M$ )}

$M$ was assumed to be 0.05 for young and 0.03 for yearlings and adult individuals (Solberg et al. 1999, 2000, 2006). The choice of $M$ was based on the very low natural mortality estimated from radio-collared moose in a number of Scandinavian populations (Solberg et al. 2005). No annual variation in $M$ and no age-specific variation in $M$ among adult females were assumed. Because survival rate for yearlings and adults is generally high and stable, the effect of these assumptions may be of minor importance in large mammals (Fowler 1987; Sæther 1997; Gaillard et al. 1998, 2000). However, because senescence has been detected after 7-9 years of age in ungulate populations (Gaillard et al. 1993, Loison et al. 1999, Festa-Bianchet et al. 2003), higher natural mortalities for older individuals may make the reconstructed population more realistic. Nevertheless, we did not have quantitative data on age-specific natural mortality rate to differentiate older individuals.

\section{Testing separate assumptions for Model $A, B$, and $C$}

Original cohort analysis. Let June (birth season) be the start of a year. Assuming that hunting is a pulse-like event that occurs in the middle of the year and that animals are subject to $M$ throughout the year, the numberat-age $\left(N_{a, y}\right)$ is given by the following equation (Pope 1972; Yatsu et al. 2002):

$$
N_{a, y}=N_{a+1, y+1} \exp (M)+C_{a, y} \exp (M / 2)
$$

The initial value for calculation by original cohort analysis was the number of individuals at oldest age $A$ $\left(N_{A, y+A-a}\right)$. We assumed that $N_{A, y+A-a}$ was equal to the number harvested at the oldest age $\left(C_{A, y+A-a}\right)$. Then, $N_{a, y}$ can be obtained by backward calculation of the number of individuals from the initial values using Equation (1).

In the original cohort analysis, number-at-age is calculable only for cohorts for which harvest-at-age data were available from $a$ to $A$ (Fig. 1a: number-at-age shaded in gray). We used harvest-at-age data from 0 to 14 years of age $(A=14)$ because the number of individuals beyond 14 age is negligibly small (Solberg et al. 1999, 2000, 2006). Thus, using data for the period 1981-2004, we were able to calculate number-at-age for all ages in cohorts alive during the period 1981-1990. Using the calculated number-at-age, $N_{a, y}$, we also calculated the age-specific hunting mortality rate $F_{a, y}$ as follows (Pope 1972; Yatsu et al. 2002):

$$
F_{a, y}=-\ln \left(1-\frac{C_{a, y} \exp (M / 2)}{N_{a, y}}\right)
$$

We tested separate assumptions for Models A, B, and $\mathrm{C}$ using calculated number-at-age and calculated agespecific hunting mortality rates for all ages in cohorts alive during the period 1981-1990. The plus-group was set to 9 years of age $(p=9)$ because pooling $C_{a, y}$ beyond that age made the harvest numbers at ages $p+$ and $p-1$ exceed zero in every year.

Assumptions for Model A. A model for rate of agespecific hunting mortality in the most recent year (Model A for $\left.\hat{F}_{a, Y}\right)$ assumes that $\hat{F}_{a, Y}$ is equal to the age-specific hunting mortality rate in the preceding year (Model A-I: Hiramatsu 2001), the arithmetic mean of age-specific hunting mortality rates during the 3 preceding years (Model A-II; Ueda et al. 2006) or during the preceding 5 years (Model A-III; Yatsu et al. 2002), taking into consideration the circumstances of harvest. These assumptions are differentiated by the period of years they represent. We also examined another model that assumes that $\hat{F}_{a, Y}$ is equal to the arithmetic mean of age-specific hunting mortality rates during the preceding 7 years (Model A-IV).

To test which assumption is most realistic, we compared the difference between an estimated value based on a model and a calculated (observed) rate of agespecific hunting mortality in year $Y(Y=1988,1989$, 1990) between four models:

$$
S S R=\left(F_{a, Y}-\hat{F}_{a, Y}\right)^{2}
$$

Assumptions for Model B. Previous studies have assumed that if there are no differences in behavior or distribution of individuals between consecutive older age classes (ages $p-1$ and $p+$ ); the rate of hunting mortality 
at age $p+$ may be assumed to be proportional to the rate of hunting mortality at age $p-1$ in all years, with a slope of $\alpha$ without an intercept. For simplicity, $\alpha$ was set at 1 (Restrepo and Powers 1991; Shepherd 1999; Hiramatsu 2001; Yatsu et al. 2002; Ueda et al. 2006):

$$
F_{9+, y}=\alpha F_{8, y}+\beta
$$

where $\alpha=1, \beta=0$ (Model B-i).

We tested Model B-i using rates of calculated hunting mortality in the last two age classes in the period 19811998.

We also modeled hunting mortality rate at age $p^{+}$ using the relationship between calculated rates of hunting mortality for the last two age classes that are calculable for early years using the original cohort analysis. Here, we introduced a simple linear-regression equation for the rate of hunting mortality at age $9+$ against that same mortality rate at age 8 :

$$
F_{9+, y}=\alpha F_{8, y}+\beta
$$

where $\alpha$ and $\beta$ were estimated from calculated rates of hunting mortality in the last two age classes, which are calculable from the original cohort analysis (Model B-ii).

We compared the estimated rate of hunting mortality at age $9+\left(\hat{F}_{9+, y}\right)$ against the calculated value $\left(F_{9+, y}\right)$ for the period 1981-1998 between Model B-i and Model Bii in order to test which is the more realistic.

Assumptions for Model C. According to Hiramatsu
(2001), the sum of number-at-age within the plus group for one year (i.e., $N_{9, y}+N_{10, y}+N_{11, y}+N_{12, y}+N_{13, y}+N_{14, y}$ ) is assumed to be equal to the sum of number-at-age within the plus group for a particular cohort (i.e., $N_{9, y}+$ $\left.N_{10, y+1}+N_{11, y+2}+N_{12, y+3}+N_{13, y+4}+N_{14, y+5}\right)$. To test the reliability of this assumption, we calculated and compared the sum of number-at-age within the plus group for one year in the period 1981-1999 against the sum of number-at-age within the plus group for corresponding cohorts.

Demonstration of cohort analysis combining three models Cohort analysis combining three models (Fig. 1b). We estimated number-at-age in the period 1981-1990 (i.e., $Y=1990)$ using $C_{a, y}$ for this period from cohort analysis combining three models. We examined the performance of eight scenarios (Model A-I, -II, -III, -IV combined with Model B-i, -ii and Model C).

For Model B-ii, we introduced a regression equation of the calculated rate of hunting mortality at age 9+ against that at age 8 in the period 1981-1984 using $C_{a, y}$ over this time span (see gray area in Fig. 1a).

We first estimated age-specific hunting mortality rate in the most recent year, $\hat{F}_{a, Y}$, and the rate of hunting mortality at age $p+$ in all years, $\hat{F}_{p^{+}, y}$, based on Models A and $\mathrm{B}$, respectively. Details of the computation of $\hat{F}_{a, Y}$ and $\hat{F}_{p^{+}, y}$ are shown in Appendix 1. Using these estimates, we computed the age-specific number of moose in the most recent year $Y$ (Hiramatsu 2001): (a)

\begin{tabular}{ccccccccccc}
\hline & \multicolumn{10}{c}{ year } \\
\cline { 2 - 11 } age & 1981 & 1982 & 1983 & 1984 & 1985 & 1986 & 1987 & 1988 & 1989 & 1990 \\
\hline 0 & 12 & 10 & 12 & 23 & 18 & 24 & 26 & 22 & 27 & 27 \\
1 & 7 & 11 & 16 & 11 & 8 & 10 & 8 & 12 & 18 & 19 \\
2 & 0 & 4 & 10 & 5 & 6 & 6 & 2 & 4 & 2 & 9 \\
3 & 12 & 7 & 6 & 4 & 7 & 7 & 5 & 8 & 9 & 8 \\
4 & 2 & 6 & 1 & 0 & 7 & 5 & 4 & 7 & 7 & 8 \\
5 & 2 & 2 & 4 & 1 & 0 & 7 & 1 & 5 & 4 & 4 \\
6 & 6 & 1 & 1 & 2 & 1 & 3 & 6 & 8 & 5 & 2 \\
7 & 2 & 1 & 5 & 0 & 5 & 1 & 0 & 4 & 9 & 3 \\
8 & 6 & 1 & 1 & 2 & 1 & 6 & 2 & 1 & 1 & 2 \\
9 & 0 & 1 & 1 & 0 & 1 & 2 & 2 & 1 & 1 & 0 \\
10 & 2 & 1 & 1 & 2 & 2 & 0 & 2 & 0 & 0 & 5 \\
11 & 0 & 2 & 0 & 1 & 0 & 1 & 1 & 0 & 0 & 3 \\
12 & 0 & 0 & 0 & 1 & 1 & 1 & 2 & 1 & 1 & 0 \\
13 & 0 & 0 & 2 & 0 & 0 & 1 & 3 & 0 & 0 & 0 \\
14 & 0 & 2 & 0 & 0 & 1 & 0 & 0 & 0 & 2 & 0 \\
\hline
\end{tabular}

(b)

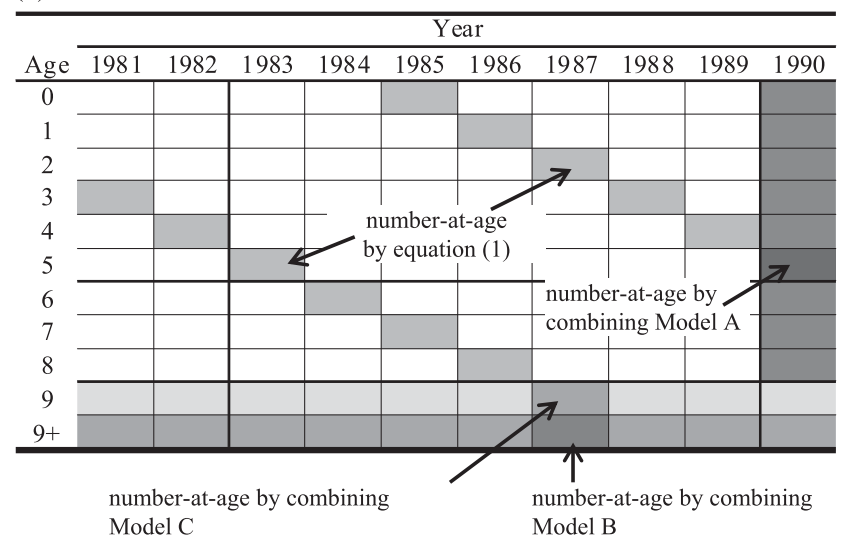

Fig. 1. (a) Harvest-at-age for female moose during the period 1981-1990. From these data, only the number-at-age shaded in gray is calculable by the original cohort analysis. (b) Cohort analysis combining three models. Number-at-age throughout the period is estimated from harvest-atage in Fig. 1a, combining three models with the original cohort analysis. Number-at-age in the most recent year $(Y)$ and number at age $p+$ in each year were computed using estimates from Models A and B applying Equation (6) and (7) and Model C applying Equation (8). Subsequently, the remainder of numbers-at-age throughout the period were computed using Equation (1). 


$$
\hat{N}_{a, Y}=\frac{C_{a, Y}}{1-\exp \left(-\hat{F}_{a, Y}\right)} \exp \left(\frac{M}{2}\right)
$$

Similarly, we computed the number of moose of age $p+$ in all years as follows (Hiramatsu 2001):

$$
\hat{N}_{p+, y}=\frac{C_{p+, y}}{1-\exp \left(-\hat{F}_{p+, y}\right)} \exp \left(\frac{M}{2}\right)
$$

We estimated the number of moose of age $p$ from the number of moose of age $p+$ using following equation, which is obtained analytically from Model C (Hiramatsu 2001):

$$
N_{p, y}=N_{p+, y}\left(1-\exp \left(-\left(F_{p+, y}+M\right)\right)\right)
$$

The remaining number-at-age throughout the period was computed by Equation (1).

To examine the performance of these scenarios, we obtained the estimation error for number-at-age:

$$
v\left(\hat{N}_{a, y}\right)=\left(\frac{N_{a, y}-\hat{N}_{a, y}}{N_{a, y}}\right)^{2}
$$

Estimation error in total population size was expressed as follows:

$$
\tau\left(\hat{N}_{\text {total }, y}\right)=\left(\frac{N_{\text {total }, y}-\hat{N}_{\text {total }, y}}{N_{\text {total }, y}}\right)^{2}
$$

\section{Robustness of the number-at-age estimate}

According to Pope (1972) and Siddeek (1982), proportional error in number-at-age $\rho\left(\hat{N}_{a, y}\right)$ of a cohort is expressed theoretically as:

$$
\rho\left(\hat{N}_{a, y}\right) \cong\left(\frac{F_{s, t}-\hat{F}_{s, t}}{\hat{F}_{s, t}}\right)\left(\exp \left(-Q_{a, y}\right)\right)
$$

where $\hat{F}_{s, t}$ is the rate of terminal hunting mortality at age $s$ in year $t$ of that cohort, i.e., the rate of age-specific hunting mortality in the most recent year $\left(\hat{F}_{a, Y}\right)$ from Model A, or the rate of hunting mortality at age $p\left(\hat{F}_{p, y}\right)$ from Models B and C. $Q_{a, y}$ is the rate of age-specific cumulative hunting mortality defined as the sum of calculated age-specific hunting mortality rates from age $a$ to $s-1$ within the cohort (i.e., $F_{a, y}+F_{a+1, y+1}+F_{a+2} \ldots+$ $F_{s-1, t-1}$; Pope 1972). Equation (11) indicates that, although the estimation error depends on the authenticity of Models A, B, and C, the extent of dependency is lower as $Q_{a, y}$ becomes larger. We calculated $Q_{a, y}$, and then examined the relationship between the estimation error in number-at-age and $Q_{a, y}$.

\section{Results}

Testing separate assumptions for Models $A, B$, and $C$ Assumption for Model A. For Models A-I through -IV, differences were not great between model-estimated values of age-specific hunting mortality rates in the most recent year $(Y=1988,1989,1990)$ and a calculated (observed) value (Fig. 2).

Assumption for Model B. The relationship between calculated rates of hunting mortality at age $9+\left(F_{9+, y}\right)$ and age $8\left(F_{8, y}\right)$ did not support Model B-i $\left(F_{9+, y}=F_{8, y}\right.$; Fig. 3). When $F_{8, y}$ was $<0.2, F_{9+, y}$ was highly variable. Conversely, when $F_{8, y}$ was $>0.2, F_{9+, y}$ appeared negatively linearly correlated with $F_{8, y}$. Coefficients of the regression relationship were statistically different from 1 for the slope $(t=-5.88, P<0.01)$ and different from 0 for the intercept $(t=6.54, P<0.01)$. Although the simple linear least-squares regression for $F_{9+, y}$ against $F_{8, y}$ was not significant (Model B-ii: $\mathrm{Y}=-0.21 \mathrm{X}+0.44$,

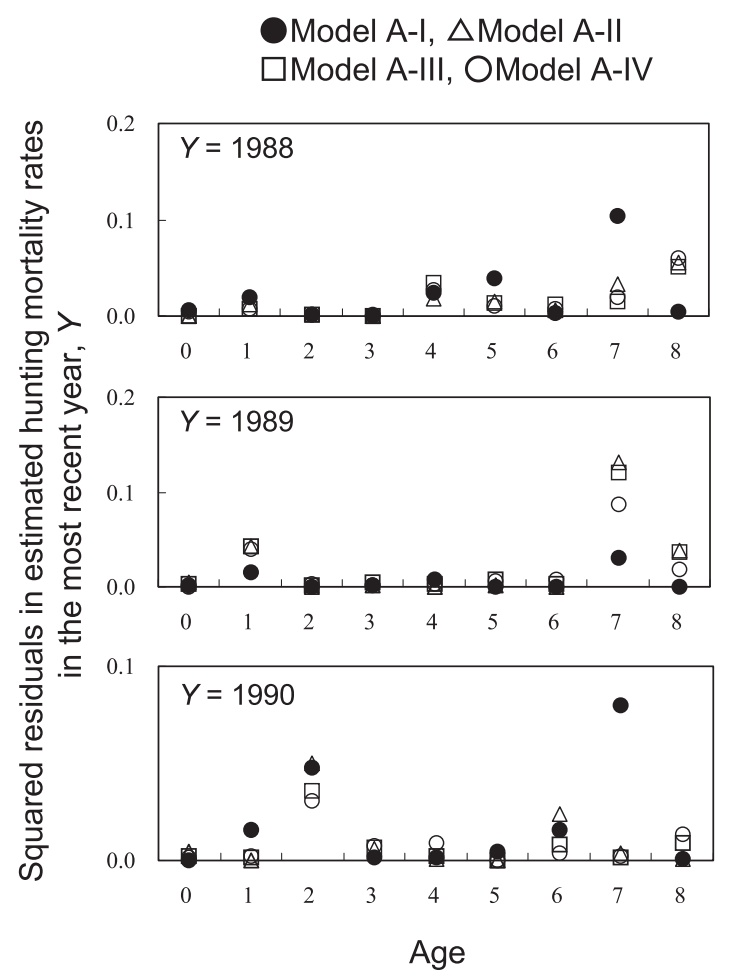

Fig. 2. Differences between estimated model values of age-specific hunting mortality rates and calculated values for the most recent year $Y(Y=1988,1989,1990)$ using four variants of Model A. Models assume (1) that age-specific hunting mortality rate is equal to the agespecific hunting mortality rate in the preceding year (Model A-I, $\mathbf{0}$ ), (2) the arithmetic mean of the age-specific hunting mortality rates in the preceding 3 years (Model A-II, $\triangle$ ), (3) 5 years (Model A-III, $\square$ ), and (4) 7 years (Model A-IV, $\bigcirc$ ). 


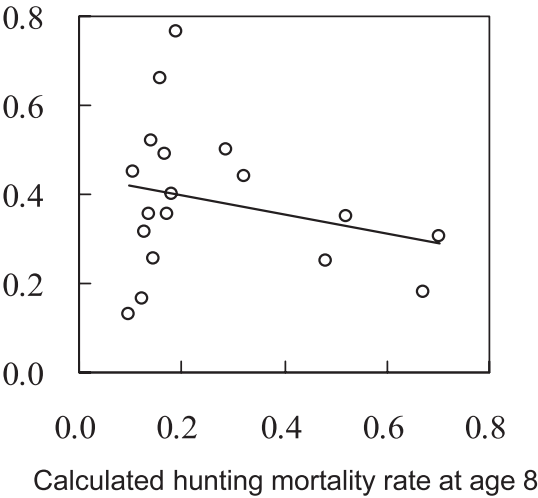

Fig. 3. Calculated hunting mortality rate at age 9+ against calculated hunting mortality rate at age 8 for the period 1981-1998. The linear regression line fitted by least squares analysis (Model B-ii) is not statistically significant $\left(\mathrm{Y}=-0.21 \mathrm{X}+0.44, r^{2}=0.06, P=0.32\right)$.

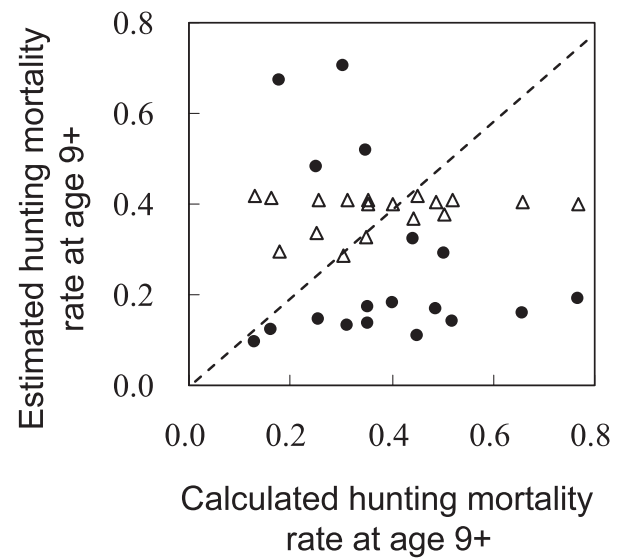

Fig. 4. Model B-i $(\bullet)$ and Model B-ii $(\triangle)$ estimated hunting mortality rates at age $9+$ against calculated hunting mortality rate at age $9+$ for the period 1981-1998. Model B-i assumes that the hunting mortality rate at age $9+$ is equal to that at age $8\left(F_{9+, y}=F_{8, y}\right)$. Model B-ii is a linear regression equation for calculated hunting mortality rate at age $9+$ against that at age $8\left(F_{9+, y}=-0.21 F_{8, y}+0.44\right)$.

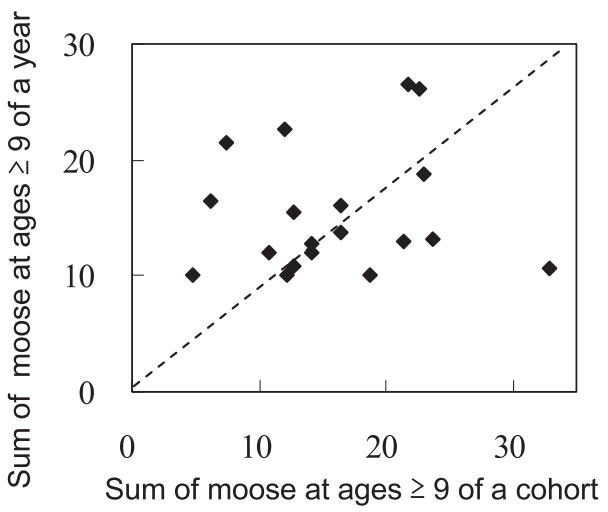

Fig. 5. Sum of moose at ages $\geq 9$ within a year against sum of moose at ages $\geq 9$ within a cohort during 1981-1999. $r^{2}=0.06, P=0.32, n=18$ ), estimated hunting mortality rates at age $9+$ from Model $\mathrm{B}$-ii were closer to the line than values estimated with Model B-i (Fig. 4).

Assumption for Model C. The estimated sum of number-at-age $\geq 9$ years old for a year was close to, but not equal to the sum of number-at-age $\geq 9$ years old calculated by cohort analysis (Fig. 5). Thus, the assumption that the sum of number-at-age within the plus group for one year is equal to the sum of number-at-age for a particular cohort was not always met.

Demonstration of cohort analysis combining three models

Estimation errors in numbers-at-age in 1990. Model A-IV estimation errors in number-at-age in 1990, were lowest among versions of Model A (Models A-I through A-IV) for six of nine age-classes (Table 1). For two of the age-classes, Models A-III and Model A-II reduced the error most. Model A -I reduced the error to the greatest extent for only one age-class. Moreover, the errors using Model A-I were larger for overall age-classes than those obtained using the other three models. Thus, errors in estimating number-at-age in 1990 generally decreased when longer runs of data were included in the model (i.e. in the sequence Model A-I to Model A-IV; Table 1).

Estimation error in the number of moose at age 9+ in all years. Model B-ii estimation errors in numbers of moose at age 9+ were much smaller than those of Model B-i for 5 of 10 years (mostly for earlier age classes) but were similar or larger for the last 4 years (Table 2).

Estimation error in the number of moose at age 9 in all years. Overall estimation errors in number of moose at age $9, v\left(\hat{N}_{9, y}\right)$, ranged up to $113 \%$ (Table 2). The error in number of moose at age 9 was not different between Model B-ii and Model B-i (Table 2).

Estimation errors in number-at-age and total population size throughout the period. We calculated estimation errors in number-at-age using a combination of Models A-IV, Model B-ii, and Model C (Table 3). Estimation errors were smaller for younger ages in earlier years. The estimation errors in number-at-age $\leq 3$ years old up to 1987 were less than $20 \%$, whereas the estimation errors in number-at-age $\geq 4$ years old and the estimation errors in number-at-age in 1988 and after were more than $20 \%$ (Table 3). Estimation errors in total population size were smaller than those of number-at-age (Table 3).

The estimation errors in total population size were larger for later years for every combination of models (Table 4). The estimation error for late years was differentiated by four variants of Model A; the error tended to 
Table 1. Estimation errors (\%) in number-at-age in 1990 using Models A-I through A-IV as a demonstration of cohort analysis combining three models. Models assume that age-specific hunting mortality rate is equal to the age-specific hunting mortality rate of the preceding year (Model A-I), the arithmetic mean of age-specific hunting mortality rates in the preceding 3 years (Model A-II), 5 years (Model A-III) and 7 years (Model A-IV)

\begin{tabular}{|c|c|c|c|c|c|c|c|c|c|}
\hline \multirow{2}{*}{ Model A } & \multicolumn{9}{|c|}{ age } \\
\hline & 0 & 1 & 2 & 3 & 4 & 5 & 6 & 7 & 8 \\
\hline I & 17 & 41 & 344 & 8 & 22 & 45 & 41 & 22 & 2 \\
\hline II & 0 & 15 & 618 & 0 & 6 & 20 & 34 & 15 & 21 \\
\hline III & 0 & 10 & 184 & 3 & 0 & 9 & 28 & 8 & 21 \\
\hline IV & 0 & 7 & 147 & 3 & 7 & 4 & 19 & 5 & 20 \\
\hline
\end{tabular}

Table 2. Model B-i and Model B-ii estimation errors (\%) in number of moose at age $9+$ and Model C estimation errors (\%) in number of moose at age 9 when combined with each version of Model B during the period 1981-1990. Model B-i assumes that the hunting mortality rate at age 9+ is equal to that at age $8\left(F_{9+, y}=F_{8, y}\right)$. Model B-ii is a linear regression equation of calculated hunting mortality.

\begin{tabular}{|c|c|c|c|c|c|c|c|c|c|c|c|}
\hline \multirow{2}{*}{ age } & \multirow{2}{*}{ Models } & \multicolumn{10}{|c|}{ year } \\
\hline & & 1981 & 1982 & 1983 & 1984 & 1985 & 1986 & 1987 & 1988 & 1989 & 1990 \\
\hline \multirow[t]{2}{*}{$9+$} & $B-i$ & 42 & 226 & 20 & 1 & 71 & 6 & 5 & 11 & 8 & 0 \\
\hline & B-ii & 0 & 3 & 1 & 3 & 0 & 1 & 169 & 33 & 27 & 3 \\
\hline \multirow[t]{2}{*}{9} & $C \& B-i$ & 1 & 1 & 11 & 16 & 72 & 6 & 89 & 54 & 30 & 0 \\
\hline & $\mathrm{C} \& \mathrm{~B}-\mathrm{ii}$ & 0 & 0 & 14 & 16 & 51 & 5 & 113 & 56 & 37 & 0 \\
\hline
\end{tabular}

Table 3. Estimation errors (\%) in number-at-age during 1981-1990 when combining Model A-IV, Model B-ii and Model C

\begin{tabular}{|c|c|c|c|c|c|c|c|c|c|c|}
\hline \multirow{2}{*}{ age } & \multicolumn{10}{|c|}{ year } \\
\hline & 1981 & 1982 & 1983 & 1984 & 1985 & 1986 & 1987 & 1988 & 1989 & 1990 \\
\hline 0 & 0 & 1 & 0 & 1 & 1 & 1 & 1 & 34 & 4 & 0 \\
\hline 1 & 6 & 0 & 1 & 0 & 3 & 1 & 3 & 2 & 66 & 7 \\
\hline 2 & 13 & 9 & 0 & 2 & 1 & 4 & 1 & 4 & 3 & 147 \\
\hline 3 & 3 & 13 & 12 & 0 & 2 & 1 & 5 & 2 & 4 & 3 \\
\hline 4 & 2 & 5 & 23 & 20 & 0 & 3 & 1 & 7 & 2 & 7 \\
\hline 5 & 4 & 2 & 8 & 26 & 20 & 0 & 4 & 2 & 13 & 4 \\
\hline 6 & 4 & 5 & 3 & 11 & 30 & 20 & 0 & 4 & 3 & 19 \\
\hline 7 & 8 & 10 & 6 & 4 & 14 & 35 & 30 & 0 & 7 & 5 \\
\hline 8 & 0 & 11 & 12 & 19 & 4 & 28 & 41 & 30 & 0 & 20 \\
\hline 9 & 0 & 0 & 14 & 16 & 51 & 5 & 113 & 56 & 37 & 0 \\
\hline $9+$ & 0 & 3 & 1 & 3 & 0 & 1 & 169 & 33 & 27 & 3 \\
\hline total & 0 & 0 & 1 & 1 & 1 & 0 & 0 & 0 & 1 & 2 \\
\hline
\end{tabular}

decrease as longer periods of data were included in the model (i.e., in the sequence Model A-IV to A-I).

\section{Robustness of the number-at-age estimate}

The relationship between proportional error in number-at-age, $\rho\left(\hat{N}_{a, y}\right)$ and age-specific cumulative hunting mortality rate $\left(Q_{a, y}\right)$ was consistent with theoretical values (Fig. 6). As $Q_{a, v}$ increased, the estimation error converged to smaller values regardless of model misspecification; the error ranged from $-64 \%$ to $81 \%$ below a $Q_{a, y}$ of 0.5 , whereas error was between $-16 \%$ and $34 \%$ above a $Q_{a, y}$ of 1.0 .

The magnitude of $Q_{a, y}$ ranged from 0.1 to 1.9 (Fig. 6). Most of the values were below 1.5 . 
Table 4. Estimation errors (\%) in total population size in the period 1981-1990 using eight different model combinations (four for Model A combined with two for Model B and Model C)

\begin{tabular}{cccccccccccc}
\hline \multirow{2}{*}{ Model A } & \multirow{2}{*}{ Model B } & \multicolumn{10}{c}{ year } \\
\cline { 3 - 11 } & 1981 & 1982 & 1983 & 1984 & 1985 & 1986 & 1987 & 1988 & 1989 & 1990 \\
\hline I & & 1 & 0 & 0 & 1 & 1 & 4 & 4 & 0 & 4 & 14 \\
II & i & 1 & 0 & 0 & 1 & 1 & 3 & 3 & 1 & 5 & 4 \\
III & 1 & 0 & 0 & 1 & 1 & 2 & 1 & 0 & 2 & 1 \\
IV & 1 & 0 & 0 & 1 & 0 & 1 & 0 & 1 & 3 & 2 \\
\hline I & & 0 & 0 & 0 & 1 & 2 & 3 & 2 & 0 & 1 & 6 \\
II & \multirow{2}{*}{ ii } & 0 & 0 & 1 & 1 & 2 & 2 & 1 & 1 & 3 & 4 \\
III & 1 & 0 & 1 & 1 & 1 & 1 & 0 & 0 & 1 & 1 \\
IV & & 0 & 0 & 1 & 1 & 1 & 0 & 0 & 0 & 1 & 2 \\
\hline
\end{tabular}

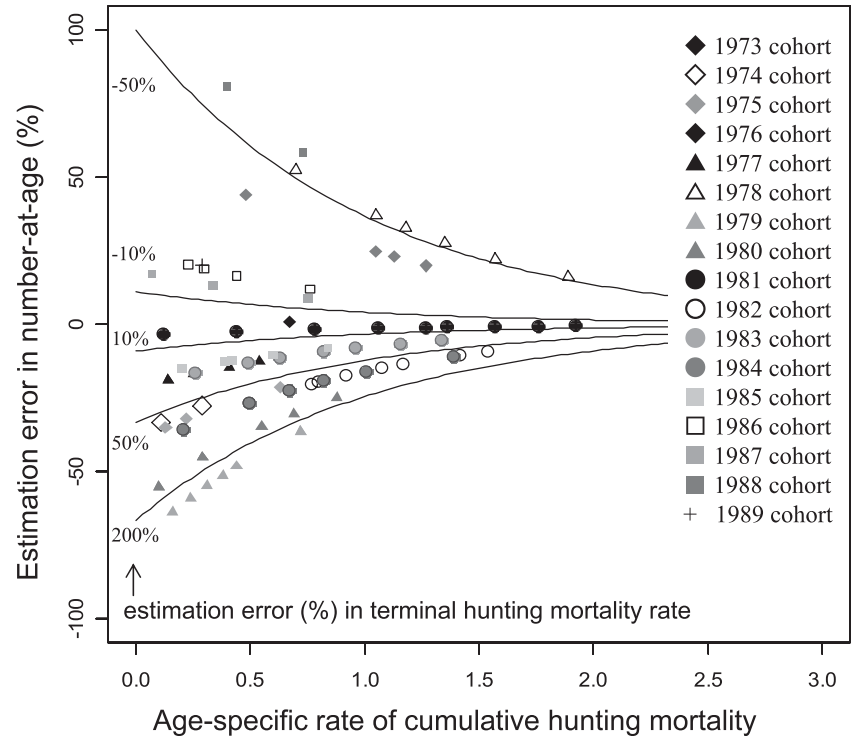

Fig. 6. Relationship between estimation error (\%) in number-at-age and age-specific rate of cumulative hunting mortality. Symbols indicate cohorts born in the period 1973-1989 (i.e., cohorts alive in the period 1981-1990). Theoretical values of Pope (1972) are shown as curves. The estimation error in terminal hunting mortality rate indicates the magnitude of misspecification by Models A, B and C.

\section{Discussion}

Exploring ways to reach realistic assumptions for Models $A, B$, and $C$

Assumption for Model A. Age-specific hunting mortality estimation errors in the most recent year, $(Y=$ $1988,1989,1990)$ were not very different among Models A-I through -IV (Fig. 2). Nevertheless, number-at-age estimation errors in $1990\left(\hat{N}_{a, 1990}\right)$ decreased with models that included age-specific hunting mortality rates spanning longer time periods (i.e., in the sequence Model A-I to Model A-IV; Table 1). It is difficult to conclude that Model A-IV is superior to other variants from this result alone. However, numbers-at-age between ages in the most recent year are indirectly connected to one another; estimation error in number-at-age at a particular age in the most recent year is subject to the magnitude of the estimation error in number-at-age at for animals one year older in the most recent year. Nevertheless, the extent of the influence becomes smaller with a Model A version using age-specific hunting mortality rates over a longer period; estimated number of moose at age $a$ in the most recent year becomes increasingly independent of the estimation error in number-at-age for $a+1$ years old with Model A variants using data over a longer period. Therefore, the best model for avoiding chain-reacting estimation errors uses the longest period of age-specific hunting mortality rates, provided there is no trend in rates of age-specific hunting mortality over years.

Assumption for Model B. The relationship between calculated rates of hunting mortality in the last two ageclasses ( 8 and 9+ years old) did not support Model B-i (Fig. 3). Model B-i might not have been realistic because of hunting regulations that include a quota system. The calculated hunting mortality rate at age $9+$ was highly variable when the rate at age 8 was $<0.2$, whereas the relationship tended to be negative when the rate at age 8 was $>0.2$ (Fig. 3). Because the total number in the harvest is limited by quota, the number of moose killed in a specific age group will always depend on the number killed in other age groups. As moose at age 8 are killed and become a smaller proportion of the total harvest, the remaining age classes should form a larger proportion of the total harvest, but this is not always the case for moose killed at age $9+$. Indeed, when moose at age 8 make up 
an increased proportion of the total harvest, the proportion of killed moose in the other age classes (including age 9+) should be smaller. This pattern will be also seen in hunting mortality rate, unless size and age structures in the population change greatly. Thus, harvest data from a quota system might limit the usefulness of Model B-i.

Estimated hunting mortality rate at age $9+\left(\hat{F}_{9+, y}\right)$ from Model B-ii was closer to the observed value than the estimate from Model B-i (Fig. 4). This is a reasonable outcome because Model B-ii was estimated by least-squares analysis based on the calculated relationship between those variables.

The estimation error for early years in the number-atage 9+ from Model B-ii was smaller than that from Model B-i (Table 2). Although estimation errors in number of moose at age 9+ from Model B-ii were considerably larger than those from Model B-i for later years (Table 2), we could find no logical reason why Model B-i would be better than Model B-ii for those years. We therefore suggest it might be better (for a more reliable estimate of hunting mortality at age $p+$ ) to construct a study-specific Model B from observational data (Model B-ii) instead of applying a previously used model (Model B-i). Although we introduced Model B-ii as a simple linear regression, the structure and shape of a model will depend on the data used for construction. Because Model B-ii can be obtained from the original cohort analysis, it is applicable to species for which age determination is accurate through the older age-classes.

Assumption for Model C. The Model C assumption was not met in this study (Fig. 5). Nevertheless, we showed that this assumption is not very influential in population estimation compared with Model A, which we discuss below.

The estimation error in number of moose at age 9 was not reduced even with application of Model B-ii (Table 2). The estimation error in number of moose at age of 9 , however, was still high.

We could not examine an alternative Model C assumption because of the difficulty in meeting the assumption of some relationship between number-at-age in a year and number-at-age within a cohort. Although Hiramatsu (2001) introduced an alternative to Model C, this alternative was analytically obtained using Model B$\mathrm{i}$, for which the assumption was not met in this study.

\section{Robustness of the number-at-age estimate}

As the age-specific cumulative hunting mortality rate,
$Q_{a, y}$, increased, the proportional error in number-at-age converged to a smaller value, indicating that the estimation was less subject to misspecification of Models A, B, and $\mathrm{C}$ (Fig. 6). Because $Q_{a, y}$ is larger for younger ages in earlier years, the number-at-age estimation error was smaller for younger ages in earlier years, but larger for older ages in earlier years and for all ages in later years (Table 3). The magnitude and pattern of total population size estimation errors were consistent with those for number-at-age estimation errors for young ages (Table 3); $>50 \%$ of the population was composed of ages $0-2$ (Appendix 2). The total population size estimation errors for late years were differentiated by comparable alternative assumptions of Model A (Table 4). This outcome occurred because number-at-age estimation errors for young ages in late years were largely subject to the reality of Model A. Thus, meeting the assumption of Model $\mathrm{A}$ is more important for the reliability of the population estimate than assumptions of Models B and C, consistent with findings of Hiramatsu (2001).

According to Pope (1972), number-at-age estimation is robust regardless of Model $\mathrm{A}, \mathrm{B}$, and $\mathrm{C}$ misspecifications when the age-specific cumulative hunting mortality rate exceeds 2.0. Although it is difficult to quantify agespecific cumulative rate of fishing mortality, the estimated rate of age-specific fishing mortality is sufficiently high to approach the critical value of 2.0 (e.g., Arcto-Norwegian cod Gadus morhua, Pope 1972; sardine Sardinops sagax, Cergole 1995; sandfish Holothuria scabra, Watanabe et al. 2005; Pacific cod Gadus macrocephalus, Ueda et al. 2006). Conversely, agespecific cumulative hunting mortality rates in our study ranged from $0.1-1.9$, and most of the values were $<1.5$ (Fig. 6), indicating that number-at-age estimation error for large terrestrial mammals will be more sensitive to model misspecification than the error for fish populations. Thus, the assumption of a reliable population size estimate must be met in the case of large terrestrial mammals, even for Model B.

Cohort analysis has been applied to aquatic species including whales and tuna, which are long-lived. While performance of models, influence of aging error in the data, and stochastic variation in population parameter estimates have been intensively studied (Shimoda et al. 2006; Mori et al. 2007), performance of Model B (assumption for plus group) has not been examined. Abundance of large terrestrial mammals in the plus group is small, around $10-10^{2}$ (Fig. 1; Ueno et al. unpublished), while it is $10^{3}$ for tuna (Nishida and Takeuchi 
1999). This suggests that estimates of plus group individuals in large terrestrial mammals will tend to be subject to demographic stochasticity in hunting mortality rate. Because the performance of Model B-ii was not markedly superior to that of Model B-i (Table 2), more simulation studies are required to examine the performance of Model B.

In our study, harvest-at-age was assumed to be measured without error. Possible measurement errors will come from the difficulties in accurate age determination or erroneous correction of harvest-at-age using a portion of harvest supplied for age determination (Restrepo and Powers 1991; Hiramatsu 1992; Yamaguchi and Matsuishi 2007). Nonetheless, accuracy of age determination for ungulates $(85.1-100 \%)$ has been verified from several studies of known-age animals (Erickson and Seliger 1969; Keiss 1969; Lockard 1972; Hamlin et al. 2000; Rolandsen et al. 2008). Jawbones of almost all harvested individuals are supplied for age determination when hunters report date, locality, sex and carcass mass (Solberg et al. 1999). Therefore, harvest-at-age measurement error in ungulates is assumed to be small; these estimates will likely be robust to measurement errors.

In contrast, Gove et al. (2002) and Conn et al. (2008) introduced a method for statistical population estimation of large terrestrial mammals when harvest-at-age is measured with error. Their method will be useful when the reported rate is low or unknown because of an insufficient reporting system or poaching. Nonetheless, some conditions must be met to apply their method. First, it is necessary to estimate a large number of hunting mortality parameters to determine number-at-age during the period of interest, which often leads to estimation failure (Radomski et al. 2005). Alternatively, practical application requires a reduction in the number of parameters by assuming a simple pattern in hunting mortality rate across age-classes, e.g., hunting mortality rate is constant over years and across ages (Gove et al. 2002), which may diverge from the actual pattern. Secondly, this method requires data on survival rate collected by radio-telemetry surveys. There are few areas where harvest-at-age data are collected concurrently with radio-telemetry surveys. We advise against applying survival estimates from natural causes and hunting in one area to another area because of great spatial variation in hunting mortality rate. The method is applicable where radio-telemetry surveys are conducted and when annual pattern in the rate of hunting mortality is similar across ages. Cohort analysis combining the three models introduced here can be applied to areas without such conditions, and will be reliable unless harvest-at-age is assumed to be greatly subject to measurement error.

To conclude, we have shown that meeting model assumptions is more important for population estimation of large terrestrial mammals than of fish. We recommend using the longest period of age-specific hunting mortality rates for modeling rates of age-specific hunting mortality in the most recent year, so long as there is no trend in age-specific hunting mortality rates over years; and we recommend using a study-specific model based on data for rates of hunting mortality at age $p+$ in all years.

Acknowledgments: We thank all the hunters in Oppland, Norway who made this study possible by their sampling and preparation of data from the moose harvested. Hino, $\mathrm{T}$ and Iijima, $\mathrm{Y}$ kindly instructed us with statistical analysis with R. We are grateful to Dyson, E and Bowyer, T who edited English and improved the manuscript. We thank two anonymous referees for critical comments and suggestions. This study was funded by the JSPS Research Fellowships for Young Scientists.

\section{References}

Cergole, M. C. 1995. Stock assessment of the Brazilian sardine, Sardinella brasiliensis, of the southeastern coast of Brazil. Scientia Marina 59: 597-610.

Conn, P. B., Diefenbach, D. R., Laake, J. L., Ternent, M. A. and White, G. C. 2008. Bayesian analysis of wildlife age-at-harvest data. Biometrics. DOI: 10.1111/j.1541-0420.2008.00987.x.

Erickson, J. A. and Seliger, W. G. 1969. Efficient sectioning of incisors for estimating ages of mule deer. Journal of Wildlife Management 33: 384-388.

Festa-Bianchet, M., Gaillard, J.-M. and Côté, S. T. 2003. Variable age structure and apparent density dependence in survival of adult ungulates. Journal of Animal Ecology 72: 640-649.

Fowler, C. W. 1987. A review of density dependence in populations of large mammals. Current Mammalogy 1: 401-441.

Fryxell, J. M., Falls, J. B., Falls, E. A., Brooks, R. J., Dix, L. and Strickland, M. A. 1999. Density dependence, prey dependence, and population dynamics of martens in Ontario. Ecology 80 $1311-1321$.

Fujise, Y., Hatanaka, H. and Ohsumi, S. 2005. What has happened to the Antarctic minke whale stocks? - A interprepation of results from JARPA - . Paper JA/J05/JR17 presented to the JARPA Review meeting, Janurary 2005. 15 pp.

Gaillard, J.-M., Delorme, D., Boutin, J.-M., Laere, G. V., Boisaubert, B. and Pradel, R. 1993. Roe deer survival patterns: a comparative analysis of contrasting populations. Journal of Animal Ecology 62: 778-791

Gaillard, J.-M., Festa-Bianchet, M. and Yoccoz, N. G. 1998. Population dynamics of large herbivores: variable recruitment with constant adult survival. Trends in Ecology and Evolution 13: 58-63.

Gaillard, J.-M., Festa-Bianchet, M., Yoccoz, N. G., Loison, A. and Toïgo, C. 2000. Temporal variation in fitness components and 
population dynamics and population dynamics of large herbivores Annual Reviews in Ecology and Systematics 31: 367-393.

Gove, N. E., Skalski, J. R., Zager, P. and Townsend, R. L. 2002. Statistical models for population reconstruction using age-at-harvest data. Journal of Wildlife Management 66: 310-320.

Gulland, J. A. 1965. Estimation of mortality rates. Annex to the Northeast Arctic working group report. International council for the exploration of the sea C.M. Doc. 3., Hamburg. 231-241.

Hamlin, K. L., Pac, D. F., Sime, C. A., DeSimone, R. M. and Dusek, G. L. 2000. Evaluating the accuracy of ages obtained by two methods for Montana ungulates. Journal of Wildlife Management 64: 441-449.

Hiramatsu, K. 1992. Possible biases in the VPA estimates of population sizes of the plus group. International commission for the conservation of Atlantic tunas (ICCAT) 39: 497-502.

Hiramatsu, K. 2001. VPA (Virtual Population Analysis). In (S. Tanaka, I. Aoki, T. Akamine and T. Ichimaru, eds.) The Report of the Project of the Establishment of the Stock Assessment System - the Textbook of Stock Assessment Method, pp. 104-128. Japan Fisheries Resource Conservation Association, Kanagawa (in Japanese).

Keiss, R. E. 1969. Comparison of eruption-wear patterns and cementum annuli as age criteria in elk. Journal of Wildlife Management 33: $175-180$.

Lebreton, J. D., Burnham, K. P., Clobert, J. and Anderson, D. R. 1992. Modeling survival and testing biological hypotheses using marked animals - a unified approach with case-studies. Ecological Monographs 62: 67-118.

Lockard, G. R. 1972. Further studies of dental annuli for aging whitetailed deer. Journal of Wildlife Management 36: 46-55.

Loison, A., Festa-Bianchet, M., Gaillard, J.-M., Jorgenson, J. T. and Jullien, J.-M. 1999. Age-specific survival and reproductive probabilities: evidence for senescence. Ecology 80: 2539-2554.

Lowe, V. P. W. 1969. Population dynamics of the red deer (Cervus elaphus L.) on Rhum. Journal of Animal Ecology 38: 425-457.

Lowe, V. P. W. and Thompson-Schwab, J. D. D. 2003. Using cohort analysis to reconstruct the size and structure of deer populations in forestry with special reference to roe deer (Capreolus capreolus L.). Forestry $76: 437-447$.

McCullough, D. R. 1979. The George Reserve Deer Herd: Population Ecology of a K-selected species. The University of Michigan Press, Michigan, USA.

Mori, M., Butterworth, D. S. and Kitakado, T. 2007. Further progress on application of ADAPT-VPA to Antarctic minke whales. Paper SC/59/IA13 presented to the IWC Scientific Committee, June 2007, Anchorage, Alaska, USA. 32 pp.

Nishida, T. and Takeuchi, Y. 1999. Stock assessment of Bigeye tune (Thunnus obesus) in the Indian Ocean based on the virtual population analysis (VPA). Indian Ocean Tuna Commission (IOTC) Proceedings no 2, pp. 195-202.

Pope, J. G. 1972. An investigation of the accuracy of virtual population analysis using cohort analysis. Research Bulletin of the International Commission for the Northwest Atlantic Fisheries 9: $65-74$.

Radomski, P., Bence, J. R. and Quinn II T. J. 2005. Comparison of virtual population analysis and statistical kill-at-age analysis for a recreational, kill-dominated fishery. Canadian Journal of Fisheries and Aquatic Sciences 62: 436-452.

Restrepo, V. R. and Powers, J. E. 1991. A comparison of three methods for handling the "plus" group in virtual population analysis in the presence of ageing errors. International commission for the conservation of Atlantic tunas (ICCAT) 35: 346-354.

Rolandsen, C. M., Solberg, E. J., Heim, M., Holmstrøm, F., Solem, M. I. and Sæther, B.-E. 2008. Accuracy and repeatability of moose (Alces alces) age as estimated from dental cement layers. European Journal of Wildlife Research 54: 6-14.
Sæther, B.-E. 1997. Environmental stochasticity and population dynamics of large herbivores: a search for mechanisms. Trend in Ecology and Evolution 12: 143-149.

Shepherd, J. G. 1999. Extended survivors analysis: An improved method for the analysis of catch-at-age data and abundance indices. ICES Journal of Marine Science 56: 584-591.

Shimoda, K., Murooka, M., Itaya, K. and Hoshino, N. 2006. Evaluation of VPA estimates of population size of brown sole Pleuronectes herzensteini in northern Hokkaido, Japan. Nippon Suisan Gakkaishi 72: 850-859 (in Japanese with English abstract).

Shirakihara, K. 1991. Sperm whale population dynamics using time series of body length data. In (K. Sakuramoto, H. Kato and S. Tanaka, eds.) Studies on Whale Stock Management, pp. 147-158. Kouseisha-kouseikaku Corporation, Tokyo, Japan.

Siddeek, M. S. M. 1982. A note on Pope's cohort analysis. ICES Journal of Marine Science 40: 209-210.

Skalski, J. R. and Millspaugh, J. J. 2002. Generic variance expressions, precision, and sampling optimization for the sex-age-kill model of population reconstruction. Journal of Wildlife Management 66: 1308-1316.

Solberg, E. J., Sæther, B.-E., Strand, O. and Loison, A. 1999. Dynamics of a harvested moose population in a variable environment. Journal of Animal Ecology 68: 186-204.

Solberg, E. J., Loison, A., Sæther, B.-E. and Strand, O. 2000. Agespecific harvest mortality in a Norwegian moose Alces alces population. Wildlife Biology 6: 41-52.

Solberg, E. J., Loison, A., Ringsby, T. H., Sæther, B.-E. and Heim, M. 2002. Biased adult sex ratio can affect fecundity in primiparous moose Alces alces. Wildlife Biology 8: 117-128.

Solberg, E. J., Grøtan, V, Rolandsen, C. M., Brøseth, H. and Brainerd, S. 2005. Change-in-sex ratio as an estimator of population size for Norwegian moose Alces alces. Wildlife Biology 11: 163172.

Solberg, E. J., Rolandsen, C. M., Heim, M., Grøtan, V., Garel, M., Sæther, B.-E., Nilsen, E. B., Austrheim, G. and Herfindal, I. 2006. Elgen i Norge sett med jegerøyne-En analyse av jaktmaterialet fra overvåkingsprogrammet for elg og det samlede sett elg-materialet for perioden 1966-2004. Norwegian Institute for Nature Research, Rapport 125, 197 pp. (in Norwegian with an English abstract).

Ueda, Y., Narimatsu, Y., Hattori, T., Ito, M., Kitagawa, D., Tomikawa, N. and Matsuishi, T. 2006. Fishing efficiency estimated based on the abundance from virtual population analysis and bottom-trawl surveys of Pacific cod Gadus macrocephalus in the waters off the Pacific coast of northern Honshu, Japan. Nippon Suisan Gakkaishi 72: 201-209 (in Japanese with English abstract).

Watanabe, K., Sugiyama, H., Sugishita, S., Suzuki, N. and Sakuramoto, K. 2005. Estimating and monitoring the stock size of sandfish Arctoscopus japonicus in the northern Sea of Japan. Fisheries Science 71: 776-783.

Yamaguchi, H. and Matsuishi, T. 2007. Effects of sampling errors on abundance estimates from virtual population analysis for walleye pollock in northern waters of Sea of Japan. Fisheries Science 73: 1061-1069.

Yatsu, A., Mitani, T., Watanabe, C., Nishida, H., Kawabata, A. and Matsuda, H. 2002. Current stock status and management of chub mackerel, Scomber japonicus, along the Pacific coast of Japanan example of allowable biological catch determinationFisheries Science 68 (Supplement I): 93-96.

Received 24 July 2008. Accepted 25 September 2008. 


\section{Appendix 1.}

Computing hunting mortality rate at age $p+\left(\hat{F}_{p^{+}, y}\right)$ and age-specific hunting mortality rate in the most recent year $Y\left(\hat{F}_{a, Y}\right)$ :

1. Give an arbitrary value to the hunting mortality rate at age $p-1$ in the most recent year $Y\left(\hat{F}_{p-1, Y}\right)$.

2. The hunting mortality rate at age $p+\left(\hat{F}_{p^{+}, y}\right)$ is computed using Model B.

3. Number of moose at ages $p+\left(\hat{N}_{p+, y}\right)$ is computed using Equation (7).

4. Number of moose at ages $p\left(\hat{N}_{p, Y}\right)$ is computed using Equation (8).

5. Number of moose at age $p-1$ in the preceding year of $Y\left(\hat{N}_{p-1, Y-1}\right)$ is computed using Equation (1).

6. The hunting mortality rates at age $p-1$ in year $Y-1\left(\hat{F}_{p-1, Y-1}\right)$ is computed using Equation (2).

7. Revert to step 2 to compute hunting mortality rates at age $p+$ in year $Y-1\left(\hat{F}_{p+, Y-1}\right)$

8. Perform this sequentially up to the first year of data.

9. Solve for $\hat{F}_{p-1, Y}$ to satisfy Model A. The value minimized the following objective function $I$ is searched by the quasi-Newton method of optimization procedure using R 2.4.1. $I=\left\{\hat{F}_{p-1, Y}-f\left(\hat{F}_{p-1, Y-1}, \hat{F}_{p-1, Y-2,,,}\right)\right\}^{2}$

10. Using $\hat{F}_{p-1, Y}$ and Model A, the age-specific hunting mortality rate in the most recent year $\left(\hat{F}_{a, Y}\right)$ is computed throughout the period in the same way as $\hat{F}_{p+, y}$.

\section{Appendix 2.}

Number-at-age during 1981-1990 calculated by the original cohort analysis.

\begin{tabular}{|c|c|c|c|c|c|c|c|c|c|c|}
\hline \multirow{2}{*}{ age } & \multicolumn{10}{|c|}{ year } \\
\hline & 1981 & 1982 & 1983 & 1984 & 1985 & 1986 & 1987 & 1988 & 1989 & 1990 \\
\hline 0 & 81 & 88 & 75 & 75 & 86 & 91 & 80 & 81 & 111 & 117 \\
\hline 1 & 40 & 66 & 74 & 59 & 49 & 64 & 63 & 51 & 55 & 80 \\
\hline 2 & 29 & 32 & 53 & 56 & 47 & 39 & 52 & 53 & 37 & 36 \\
\hline 3 & 44 & 28 & 27 & 42 & 50 & 40 & 33 & 49 & 48 & 34 \\
\hline 4 & 16 & 30 & 21 & 20 & 37 & 41 & 32 & 27 & 39 & 37 \\
\hline 5 & 15 & 14 & 24 & 19 & 20 & 29 & 35 & 27 & 19 & 31 \\
\hline 6 & 18 & 13 & 11 & 19 & 17 & 19 & 21 & 33 & 21 & 15 \\
\hline 7 & 12 & 11 & 11 & 10 & 17 & 15 & 15 & 15 & 25 & 15 \\
\hline 8 & 12 & 10 & 10 & 6 & 9 & 11 & 14 & 15 & 10 & 15 \\
\hline 9 & 2 & 6 & 8 & 9 & 4 & 8 & 5 & 11 & 13 & 9 \\
\hline $9+$ & 12 & 16 & 18 & 22 & 20 & 22 & 21 & 22 & 32 & 35 \\
\hline total & 280 & 308 & 325 & 328 & 351 & 371 & 366 & 372 & 398 & 415 \\
\hline
\end{tabular}

